\title{
SUPPLEMENTARY MATERIAL TO MANUSCRIPT: NEW TIME SAMPLING STRATEGY FOR THE ESTIMATION OF THE PARAMETERS IN DRSM MODELS
}

\section{Yachao Dong1, Christos Georgakis1 *, Jason Mustakis2, and Jonathan P. McMullen3}

1 Systems Research Institute, Tufts University, Medford MA 02155, USA

2 Pfizer Worldwide R\&D, Groton, CT 06340, USA

3 Merck \& Co., Inc., Process Research and Development, Rahway, NJ 07065, USA

* Corresponding Author: Christos.Georgakis@Tufts.edu 


\title{
SUPPLEMENTARY MATERIALTO MANUSCRIPT:
}

\author{
NeW Time Sampling Strategy for the Estimation \\ OF THE PARAMETERS IN DRSM MODELS \\ Yachao Dong ${ }^{1}$, Christos Georgakis ${ }^{1 *}$, Jason Mustakis ${ }^{2}$, \\ and Jonathan P. McMullen ${ }^{3}$ \\ ${ }^{1}$ Systems Research Institute, Tufts University, Medford MA 02155, USA \\ 2 Pfizer Worldwide R\&D, Groton, CT 06340, USA \\ ${ }^{3}$ Merck \& Co., Inc., Process Research and Development, Rahway, NJ 07065, USA \\ *Corresponding Author: Christos.Georgakis@Tufts.edu
}

\section{Code of "StudyMatrix.m"}

This is a Matlab m-file routine for studying matrix $B, U V, D D$ values for different strategies with different number of samples. Table 1 and Figure 2 are obtained based on these calculations.

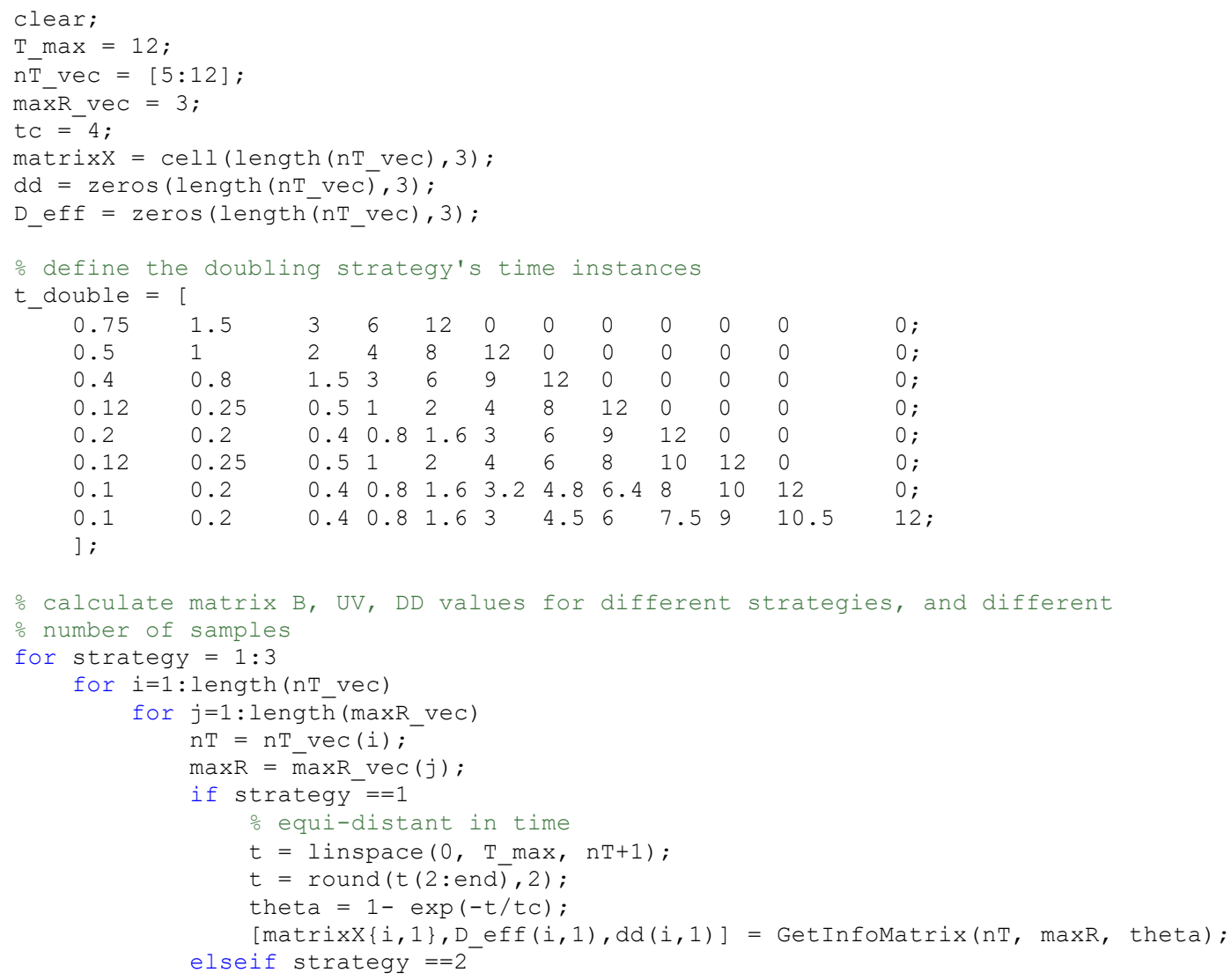




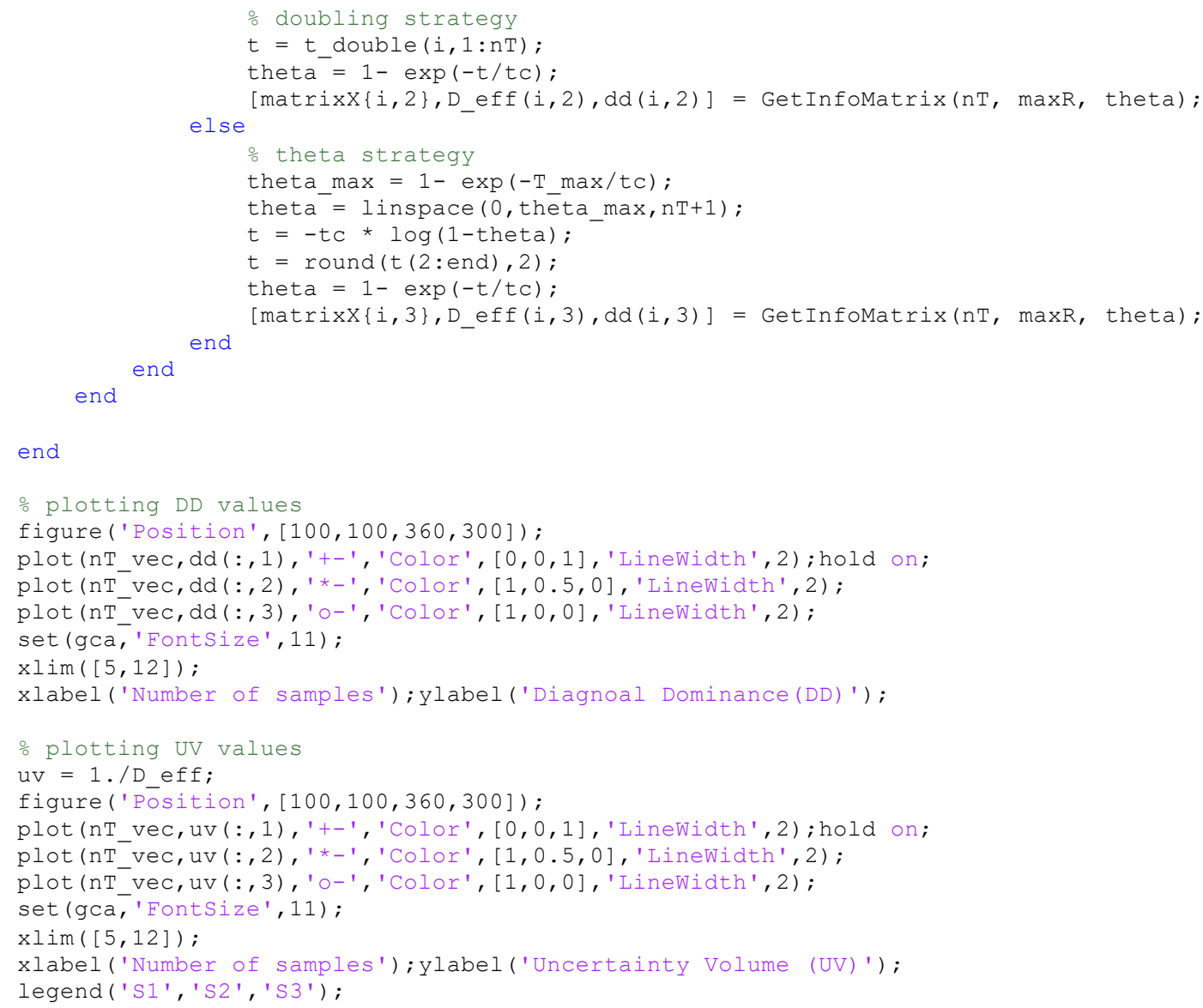

\section{Code of "PlotMatrix.m"}

This is a Matlab m-file routine for plotting matrix B with different strategies, for $\mathrm{R}=5$ and 12 samples. Figure 1 is obtained based on these calculations.

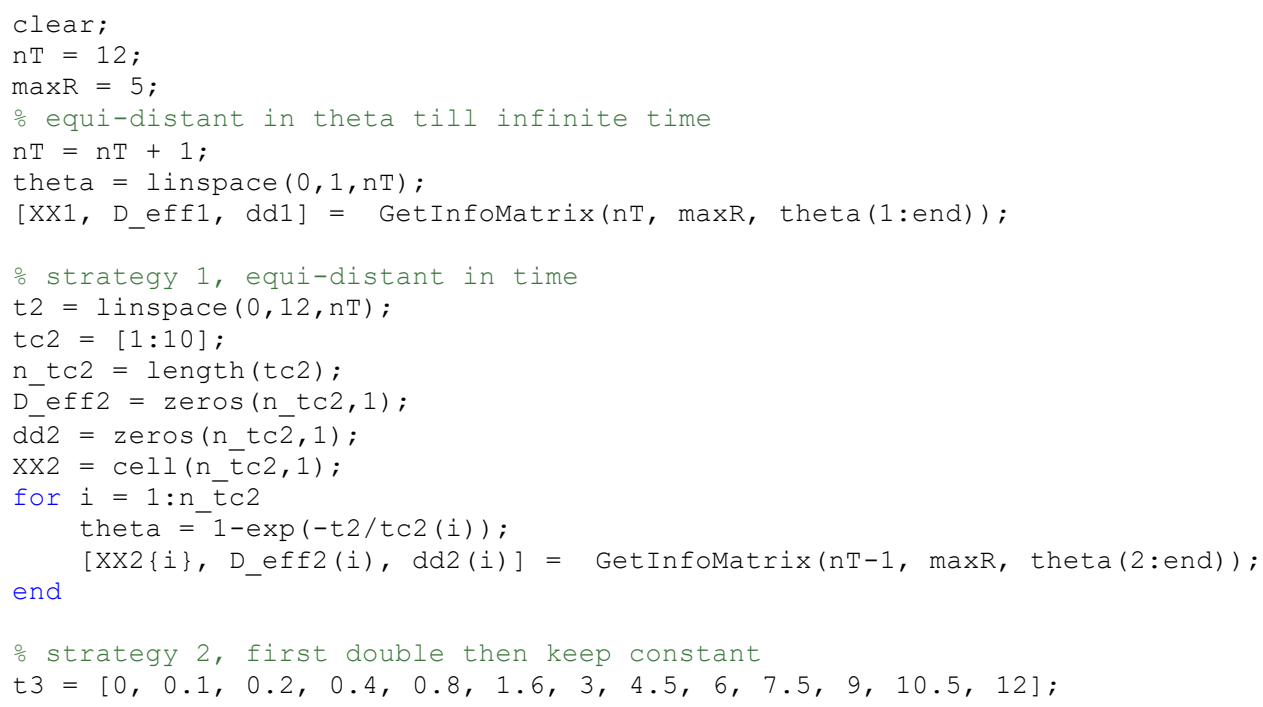




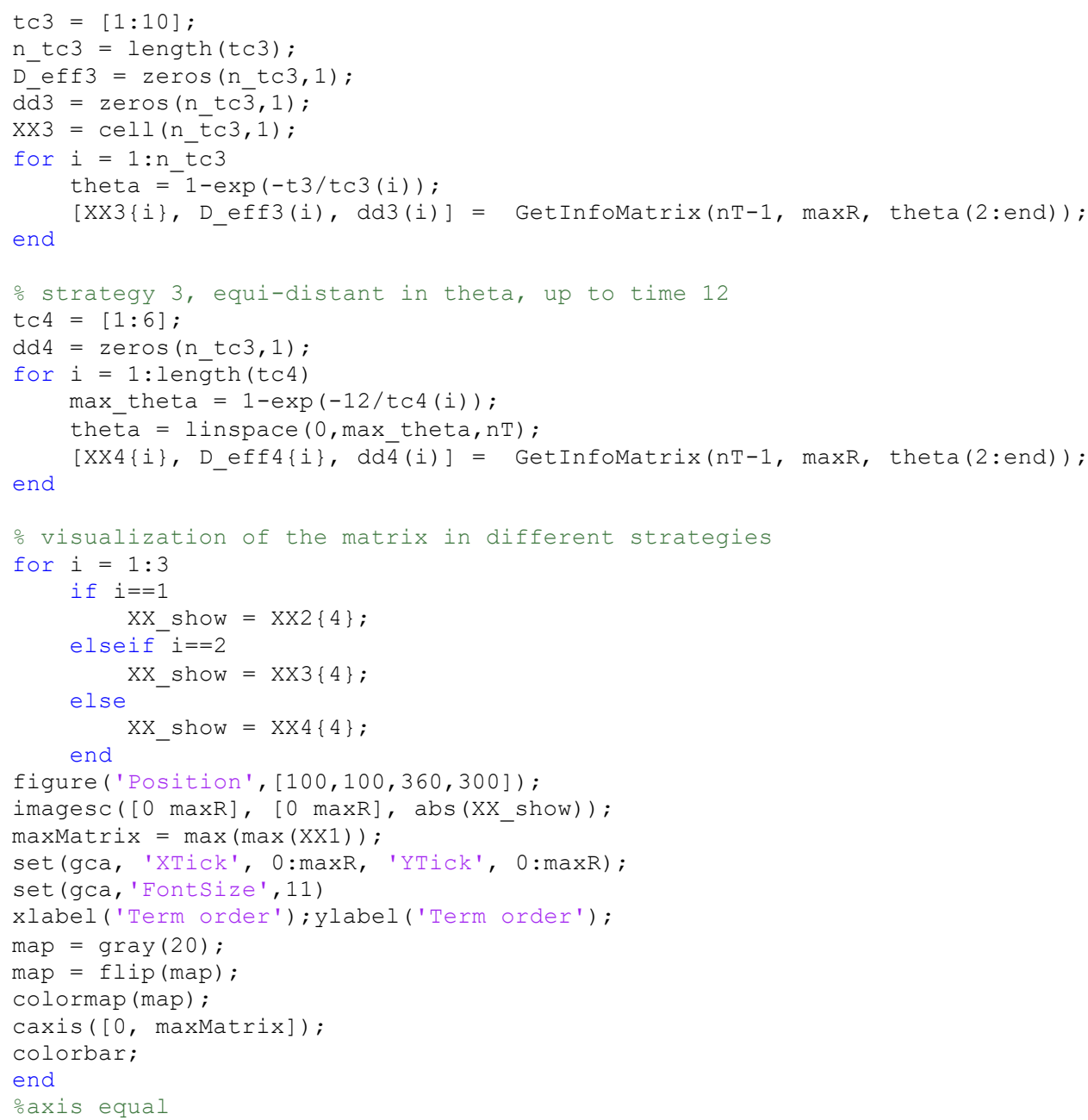

\section{Code of "GetInfoMatrix.m"}

This is a Matlab m-file routine for calculating matrix B and the corresponding values of $D$ efficiency and Diagonal Dominance. This routine is called by the code in sections 1 and 2 in this document.

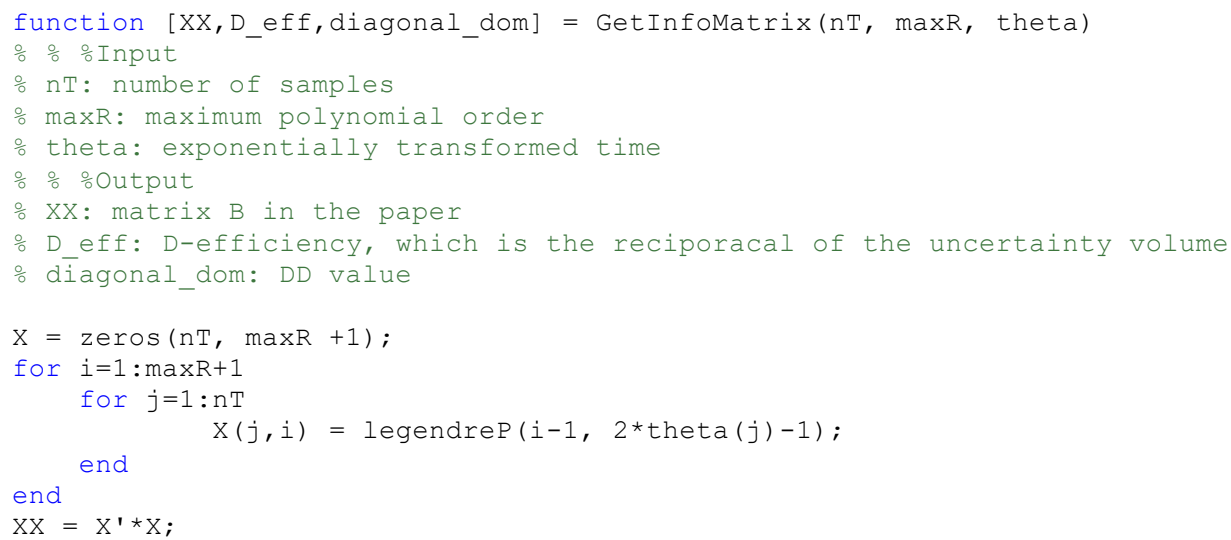




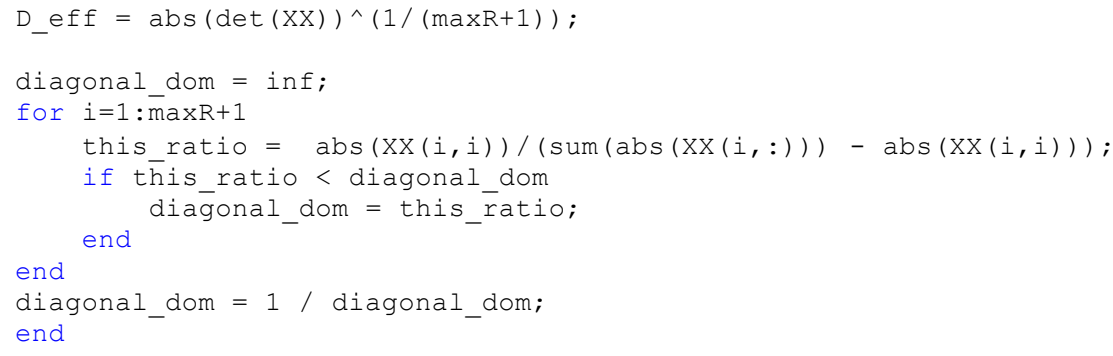

